\title{
Usefulness of a fluorescence visualization system for the detection of oral precancerous and early cancerous lesions
}

\author{
YUICHI OHNISHI $^{1}$, TOMOKO FUJII ${ }^{1}$, YOSHIHIDE UGAKI ${ }^{1}$, HIROKI YASUI $^{1}$, \\ MASAHIRO WATANABE ${ }^{1}$, SUGURU DATEOKA ${ }^{2}$ and KENJI KAKUDO ${ }^{1}$ \\ ${ }^{1}$ Second Department of Oral and Maxillofacial Surgery, Osaka Dental University, Osaka 573-1121; \\ ${ }^{2}$ Department of Dentistry for Disability and Oral Health, Osaka Dental University Hospital, Osaka 540-0008, Japan
}

Received January 3, 2016; Accepted March 10, 2016

DOI: $10.3892 / o r .2016 .4776$

\begin{abstract}
Early detection of precancerous and early cancerous lesions could greatly reduce both the mortality and morbidity of oral cancer. The objective of this study was to analyze a fluorescence visualization (FV) system for the detection of precancerous and early cancerous lesions in rat tongue carcinogenesis and human oral cancerous lesions using for the first time a 4NQO rat model and human tissue. Based on the results from the rat tongue carcinogenesis model, under direct FV, the normal oral mucosa emitted various shades of pale green autofluorescence. In the precancerous and early cancerous cases, the lesion appeared as an irregular dark area. Histological examination of the lesions showed that the VELscope system had a sensitivity of $95 \%$ and specificity of $100 \%$ in discriminating normal mucosa from dysplasia/ carcinoma in situ (CIS) or invasive carcinoma. The proliferating cell nuclear antigen (PCNA) protein level was gradually increased with progression of carcinogenic transformation. Furthermore, the results of PCNA and FV loss (FVL) were correlated. Next, results from 17 patients were also presented. Histological examination of the lesions showed that the VELscope system had a sensitivity of $95 \%$ and specificity of $100 \%$ in discriminating normal mucosa from severe dysplasia/ CIS or invasive carcinoma. There were no normal epithelium cells in any of the FVL regions. Furthermore, to clarify the usefulness of FV compared to vital staining with iodine, we investigated the surgical margins of early oral squamous cell carcinoma (OSCC) tissues and compared the FVL and iodine unstained area (IU). The percentage of various types
\end{abstract}

Correspondence to: Dr Yuichi Ohnishi, Second Department of Oral and Maxillofacial Surgery, Osaka Dental University, 8-1 Kuzuhahanazonocho, Hirakata, Osaka 573-1121, Japan

E-mail: onishi_yu_ku@msn.com

Abbreviations: FV, fluorescence visualization; CIS, carcinoma in situ; SCC, squamous cell carcinoma; FVR, fluorescence visualization retained; FVL, fluorescence visualization loss; IU, iodine unstained area

Key words: precancerous lesions, early cancerous lesions, VELscope, fluorescence visualization, squamous cell carcinoma of dysplasia were almost equal when comparing the FVL and IU. These results suggest that this direct FV device has the potential for simple, cost-effective screening, detection and margin determination of oral precancerous and early cancerous lesions.

\section{Introduction}

Squamous cell carcinoma (SCC) is the most frequent type of cancer in the oral and maxillofacial region, and its metastatic and invasive abilities result in a poor prognosis $(1,2)$. Standard care for oral cancer includes a combination of surgery, radiation and chemotherapy. Although cancer treatment is progressing substantially, the survival rate of patients with oral cancer has not changed over the past 30 years (3). Early detection of premalignant oral mucosal abnormalities and SCC is preferred because early diagnosis and appropriate treatment decrease patient morbidity and improve survival (4). The clinical symptoms of precancerous lesions and early oral squamous cell carcinoma (OSCC) are varied and may be misdiagnosed as other conditions, including mucosal inflammation, hyperkeratosis, or traumatic ulceration.

Autofluorescence is one potential technique that may be used to facilitate the visualization and detection of oral precancerous and early cancerous lesions. As early as 1924, it was discovered that the autofluorescence of tissues could potentially be used for cancer detection. Autofluorescence works on the principle that certain biofluorophores present within the tissue become fluorescent on excitation with a light source of suitable wavelength $(400-460 \mathrm{~nm})$. However, diseased tissues exhibit FV loss (FVL) due to breakdown in the dispersion of these biofluorophores, and appear darker in color. Recently, fluorescence imaging studies for the early detection of oral cancer have used indirect visualization using either photographic film or a sensitive charge-coupled device (CCD) camera. De Veld et al provide a comprehensive review of in vivo autofluorescence spectroscopy and imaging for oral oncology (5). Onizawa et al $(6,7)$ used a custom UV-flash photographic system to record porphyrin-like fluorescence in the oral resin. Fluorescence was energized by the $360-\mathrm{nm}$ spectral peak of a flash lamp and fluorescence was recorded on photographic film using a 480 -nm long-pass filter. The authors reported $91 \%$ sensitivity and $84 \%$ specificity for identification 
of benign from cancerous lesions. More recently, Svistun et al reported a system for the direct visualization of oral region fluorescence (8). In their system, excitation light was provided by a handheld illuminator and tissue fluorescence was observed along an axis slightly tilted from the illumination axis using special glasses. Realized tumor margins, as decisioned from the fluorescence images and not observed directly through the viewing glasses, were correlated with histology. The sensitivity and specificity were 91 and $86 \%$ for the identification of normal tissue from malignant lesions at the best energization wavelength.

The interaction of light with tissue has generally been found to best show changes in the structure and metabolic properties of the areas optically sampled. Concretely, the loss of autofluorescence is trusted to reveal a complex mixture of changes to essential tissue fluorophore apportioning. In this way, structural changes in tissue morphology correlated with malignant development in both the epithelium and lamina propria lead to increased absorption of light, which in turn, decreases and corrects the detectable autofluorescence (8-11).

Recent auxiliary screening technologies may allow clinicians to detect premalignant and early cancerous lesions. The technology is based on the prerequisite that normal cells fluoresce when exposed to fluorescent light, whereas abnormal cells will absorb fluorescent light and appear dark. We investigated the value of this device to draw field changes in autofluorescence around malignant lesions by determining and comparing the histopathologic changes in margins that retained normal fluorescence visualization (FVR) with those margins that showed a loss of FV (FVL). The objective of this study was to investigate the value of this device using rat tongue carcinogenesis and human oral precancerous and early cancerous lesions. This is the first such study to use this rat model.

\section{Materials and methods}

Animals. A total of 90 six-week-old male Sprague-Dawley rats (weighing from 200 to $250 \mathrm{~g}$; Clea Japan, Inc., Tokyo, Japan) were kept in an animal room maintained at a constant temperature of $24 \pm 0.5^{\circ} \mathrm{C}$. The tongues of 80 animals of the animals were rubbed with cleanser on the first day and a solution of 50 ppm 4-nitroquinoline 1-oxide (4NQO) (Nacalai Tesque Inc., Kyoto, Japan) was placed in their drinking water for 16 weeks. The remaining 10 animals were used as controls. All experimental protocols involving animals were reviewed and approved by the Animal Committee of Osaka Dental University (Osaka, Japan) and conformed with procedures described in the Guiding Principles for the Use of Laboratory Animals.

Tissue preparation. The rats were sacrificed when their tongues showed a whitened appearance with red areas and papillary lesions. In preparation for immunohistochemical staining for proliferating cell nuclear antigen (PCNA), the resected tongue tissues were fixed overnight in $4 \%$ paraformaldehyde (PFA). The specimens were embedded in paraffin, sectioned at a thickness of $4 \mu \mathrm{m}$ at right angles to the mucosal surface, and mounted on silane-coated slides. Subsequently the resected tongue tissues were immediately frozen at $-80^{\circ} \mathrm{C}$ for molecular biological examination.

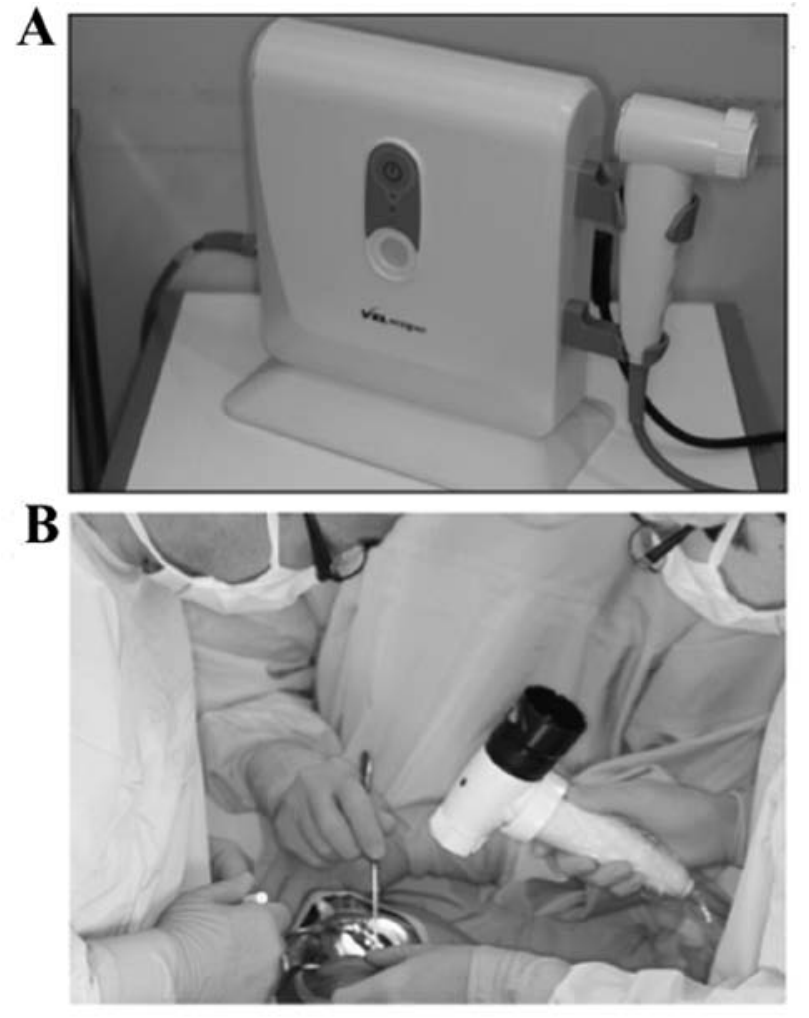

Figure 1. The VELscope system. (A) The VELscope system and (B) its clinical use.

Histopathological observation. Immunohistochemistry was performed to examine the expression of PCNA. After deparaffinization with xylene, the sections were rehydrated with $100 \%$ alcohol and washed in distilled water. Endogenous peroxidase activity was blocked by incubating the sections with $3 \% \mathrm{H}_{2} \mathrm{O}_{2}$ in methanol for $15 \mathrm{~min}$ at room temperature. Slides were incubated with monoclonal mouse antibody against human PCNA (PC10; Dako, Glostrup, Denmark) at a dilution of 1:50 at $4^{\circ} \mathrm{C}$ overnight. The sections were washed in phosphate-buffered saline (PBS) and incubated with the secondary antibody [biotinylated anti-mouse immunoglobulin (Ig)G, or anti-rabbit IgG] at room temperature $30 \mathrm{~min}$. After washing in PBS for $5 \mathrm{~min}$, the sections were stained with 3,3'-diaminobenzidine for $5 \mathrm{~min}$, washed in distilled water, counterstained with hematoxylin and eosin (H\&E) and mounted. We observed H\&E-stained and immunohistochemically stained PCNA sections at both regions. Features of the staining and amount of PCNA immunoreactivity were evaluated for each region. A scoring percentage was used in which the mean percentage of positive tumor cells was determined in $\geq 3$ random fields at x200 magnification in each section.

VELscope. This system is a simple manual device developed by LED Medical Diagnostics (Burnaby, BC, Canada) in association with scientists of the British Columbia Cancer Agency (BCCA; Vancouver, BC, Canada) (Fig. 1). It detects the loss of fluorescence in visible and non-visible high-risk oral lesions by applying direct fluorescence. The loss of fluorescence reflects a complex mixture of alterations to the intrinsic tissue distribution of fluorophores $(12,13)$. The VELscope integrates four key elements: illumination, sophisticated filtering, natural 
tissue fluorophores, and the power of human optical and neural physiology. The VELscope has the ability to surpass many of the restrictions presented by conventional methods for screening, and aid in the detection of mucosal lesions including precancerous and cancerous lesions. The VELscope illuminates tissues with specific wavelengths that interact with and provide metabolic and biochemical information concerning the cells just beneath the surface. This provides clinicians the capability to detect early biochemical changes before they become apparent, and therefore detect lesions earlier in the disease process. This system consists of a source of light that emits a wavelength of 400 to $460 \mathrm{~nm}$ and a manual unit for direct visualization. Under this light, normal oral mucosa gives off a green auto-fluorescence, whereas abnormal mucosa absorbs the fluorescent light and appear dark $(11,14)$. Therefore, early biochemical changes are discovered before their more obvious appearance, permitting the early detection of pathological lesions. Reported sensitivity values range from 97 to $98 \%$ and specificity from 94 to $100 \%(10,12,15)$. Under direct FV, normal oral mucosa presented various shades of pale green autofluorescence. Clinical lesions that retained normal green autofluorescence under FV were defined as FV retained (FVR). Tissues that showed a reduction in the normal pale green color and appeared as dark patches were classified as FVL. This distinction involved a comparison of the lesion site with both adjacent tissues and, as an anatomic control, with tissues on the contralateral side. Photographs of tissue fluorescence were acquired using illumination from the FV device and a digital single lens reflex camera with a long-pass filter. The camera was prepared with a macro lens and a ring flash for white-light images.

Patients. Twenty patients with biopsy-confirmed primary cancer of the oral region were selected for the study. Enrollment criteria included the presence of early stage disease (T0-T2) scheduled for surgical excision with the aim to treatment. Tumor staging of the surgical specimens determined that 6 were carcinomas in situ (CIS; stage 0), 13 were stage I invasive SCC and 1 was stage II invasive SCC. The majority of the tumors were from the tongue (15 of $20,75 \%$ ), while 2 cases were from the floor of the mouth, 1 from the gingiva, and 2 from the buccal mucosa.

Tissue sampling and histological assessment. After resection, a total of 60 samples were obtained from the tumor margins in each of three regions (anterior, posterior and medial). All samples were fixed in formalin and presented for histopathological assessment by pathologists who had no knowledge of the FV status. The tumor and its surrounding area were subjected to vital staining. Before the staining, the lesion was rinsed with water and dried. Iodine staining was performed using $3 \%$ iodine solution for $20 \mathrm{sec}$. Images of the lesion were captured $\sim 2$ min after the iodine application. The surgical margin was determined to be $\sim 3 \mathrm{~mm}$ outside the unstained area.

Statistical analysis. The Mann-Whitney U test was used to assess the statistical significance of differences between samples. P-values $<0.05$ were considered to indicate statistically significant results.
Table I. Correlation of direct FV results with lesion histopathology in the rat model.

Normal Dysplasia and CIS Invasive SCC

\begin{tabular}{lccc}
\hline No. of lesions & 10 & 12 & 10 \\
FVR & $10(100 \%)$ & $1(8 \%)$ & $0(0 \%)$ \\
FVL & $0(0 \%)$ & $11(92 \%)$ & $10(100 \%)$ \\
\hline
\end{tabular}

FV, fluorescence visualization; CIS, carcinoma in situ; SCC, squamous cell carcinoma; FVR, FV retained; FVL, FV loss.

\section{Results}

\section{Rat carcinogenesis model}

Analysis of the 80 experimental rats. White, red or papillary lesions appeared in 22 of the 80 experimental rats. Histologically, 7 were dysplasia, 5 were CIS and 10 were SCC. The remaining 58 rats had no lesions on the lateral edge of their tongue.

Normal tissues. Under direct FV, the normal oral mucosa emitted various shades of pale green autofluorescence (Fig.2A). Clinical lesions that retained normal green autofluorescence under direct FV were classified as lesions with FVR.

Dysplasia cases. In the VELscope image (Fig. 2B) this lesion appeared as an irregular, dark area. This area was confirmed by biopsy to be dysplasia. Tissues that showed a distinct reduction in the normal pale green and appeared as dark green to black were classified as FVL.

CIS cases. In the VELscope image (Fig. 2C) this lesion appeared as an irregular, dark area. This area was found to be severe dysplasia. Tissues that showed a distinct reduction in the normal pale green and appeared as dark green to black were classified as FVL.

SCC cases. In the SCC image (Fig. 2D) this lesion appeared as clear, dark area. This area was confirmed to be SCC. This assessment involved a comparison of the lesion site with both adjacent tissue and, as an anatomical control, with tissue on the contralateral side.

Immunohistochemical analysis. PCNA-positive cells were prominent in the parenchyma of SCC and in the basal layer of CIS and dysplasia. The PCNA-positive rate is shown in Fig. 3. PCNA-positive cells generally were increased with the progression of tumors. A significant increase in the PCNA-positive rate was observed between the control and dysplasia groups $(\mathrm{P}<0.01)$.

Pathology. As shown in Table I, of the 32 samples, 11 were FVR, and 21 were FVL. None of the 10 samples with a normal histological diagnosis showed FVL, whereas $92 \%$ of precancerous lesions (dysplasia and CIS) and 100\% of invasive SCC showed FVL. 
A

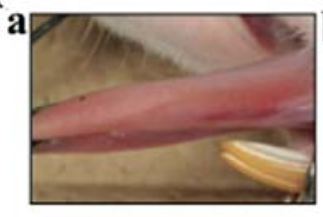

C

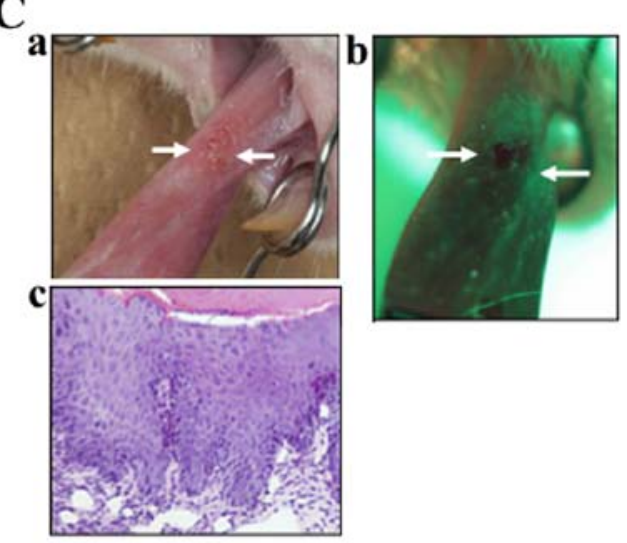

B
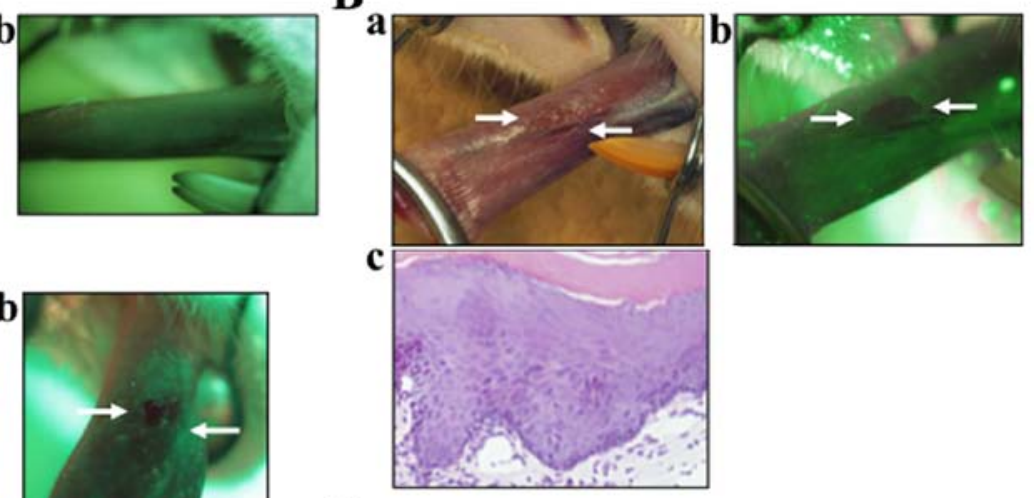

D
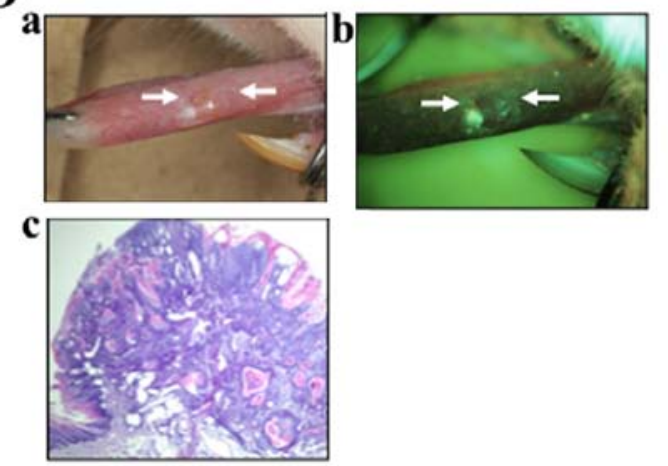

Figure 2. Fluorescence visualization (FV) in rat tongue carcinogenesis. (A) Normal tissue. White-light image is shown on the left (a) and a fluorescence image on the right (b). The images illustrate normal mucosa of the ventral tongue. Healthy tissue exhibited a pale-green fluorescence (b). (B) A case of dysplasia. The margins were confirmed to have severe dysplasia (a). Arrow in (b) indicates FV loss (FVL). The histology is shown in (c). (C) A case of carcinoma in situ (CIS). The margins were confirmed to have severe dysplasia (a). Arrow in (b) indicates FVL. The histology is shown in (c). (D) A case of squamous cell carcinoma (SCC). Arrows in (b) indicate the region of FVL which was confirmed to be SCC. The histology is shown in (c).

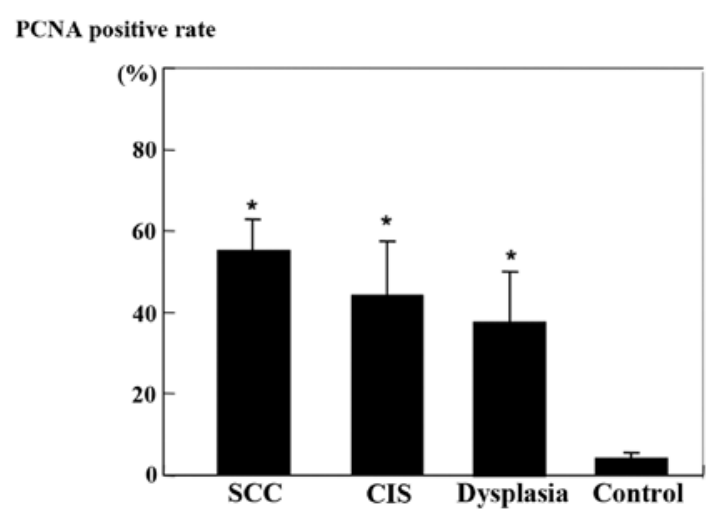

Figure 3. Expression level of proliferating cell nuclear antigen (PCNA) at various histopathological stages. A significant increase in the PCNA-positive rate was observed between the control and dysplasia groups. Data are presented as the mean $\pm \mathrm{SD}$ (Mann-Whitney $\mathrm{U}$ test; $\left.{ }^{*} \mathrm{P}<0.05\right)$.

\section{Patients}

Direct $F V$. Under direct $\mathrm{FV}$, normal oral mucosa reflects various shades of pale green autofluorescence. Clinical lesions that retained normal green autofluorescence under direct FV were classified as lesions with FVR. Tissues that showed a distinct reduction in the normal pale green and appeared as dark green to black were classified as FVL. This assessment involved a comparison of the lesion site with both adjacent tissue and, as an anatomical control, with tissue on the contralateral side.
Table II. Correlation of direct FV results with the lesion histopathology of the patients.

Normal Dysplasia and CIS Invasive SCC

\begin{tabular}{llcc}
\hline No. of lesions & \multicolumn{1}{c}{7} & 39 & 14 \\
FVR & $7(100 \%)$ & $4(10 \%)$ & $1(7 \%)$ \\
FVL & $0(0 \%)$ & $35(90 \%)$ & $13(93 \%)$ \\
\hline
\end{tabular}

FV, fluorescence visualization; CIS, carcinoma in situ; SCC, squamous cell carcinoma; FVR, FV retained; FVL, FV loss.

Normal tissues. Under direct FV, the normal oral mucosa reflected various shades of pale green autofluorescence (Fig. 4A). Clinical lesions that retained the normal green autofluorescence under direct FV were classified as lesions with FVR.

Precancerous cases. In the VELscope image, the lesion appeared as an irregular, dark area. This area was confirmed by biopsy to be dysplasia. Tissues that showed a distinct reduction in the normal pale green and appeared as dark green to black were classified as FVL (Fig. 4B).

Early SCC cases. In the early SCC image (Fig. 3C), the lesion appeared as an irregular, dark area. This area was found to be severe dysplasia. Tissues that showed a distinct reduction 
A

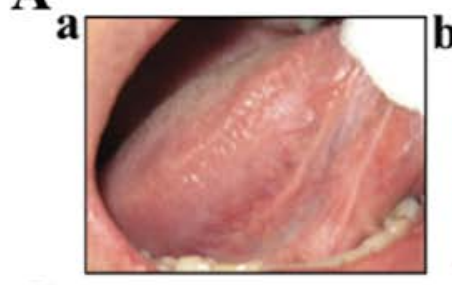

C

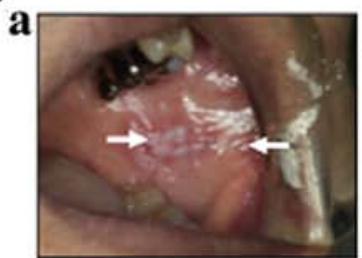

C

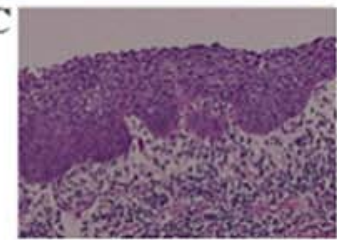

B
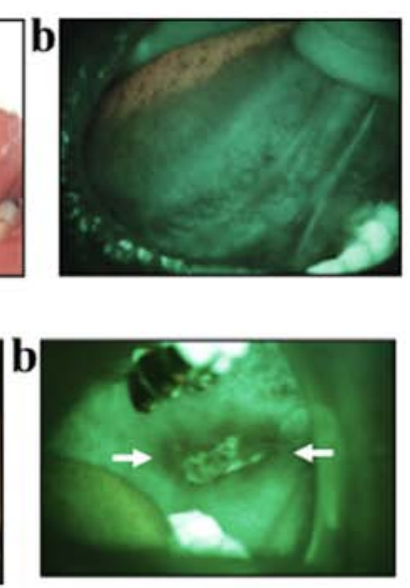

D
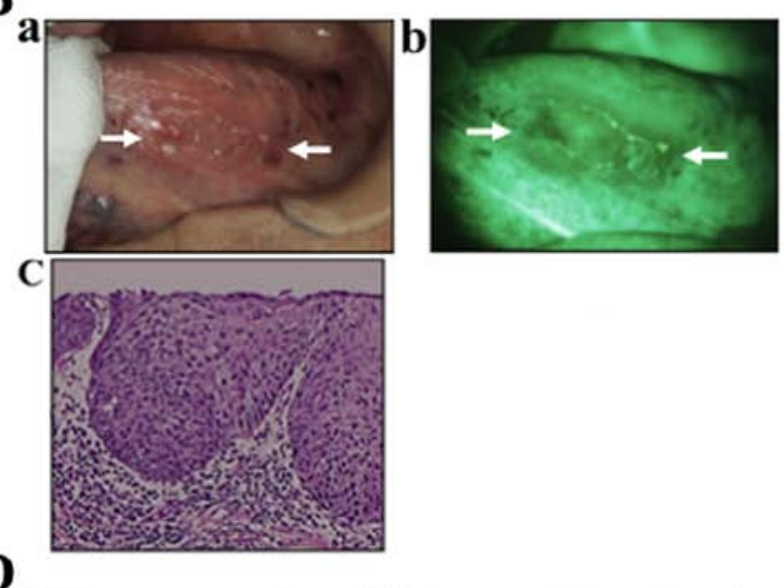

a

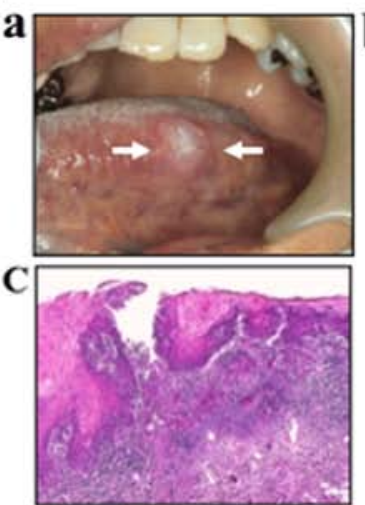

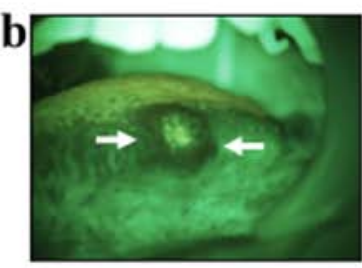

Figure 4. Fluorescence visualization (FV) in the patients. (A) Images of a normal case. White-light images are presented on the left (a) and fluorescence images on the right (b). The images illustrate normal mucosa of the ventral tongue. Healthy tissue had a pale-green fluorescence (b). (B) Image of a carcinoma in situ (CIS) case. The margin was confirmed to be severe dysplasia (a). Arrow in (b) indicates FV loss (FVL). The histology is shown in (c). (C) Image of an early squamous cell carcinoma (SCC) case. The margin was confirmed to be severe dysplasia (a). Arrow in (b) indicates FVL. The histology is shown in (c). (D) Image of a SCC case (a). Arrow in (b) indicates FVL which was confirmed to be SCC. The histology is shown in (c).

A
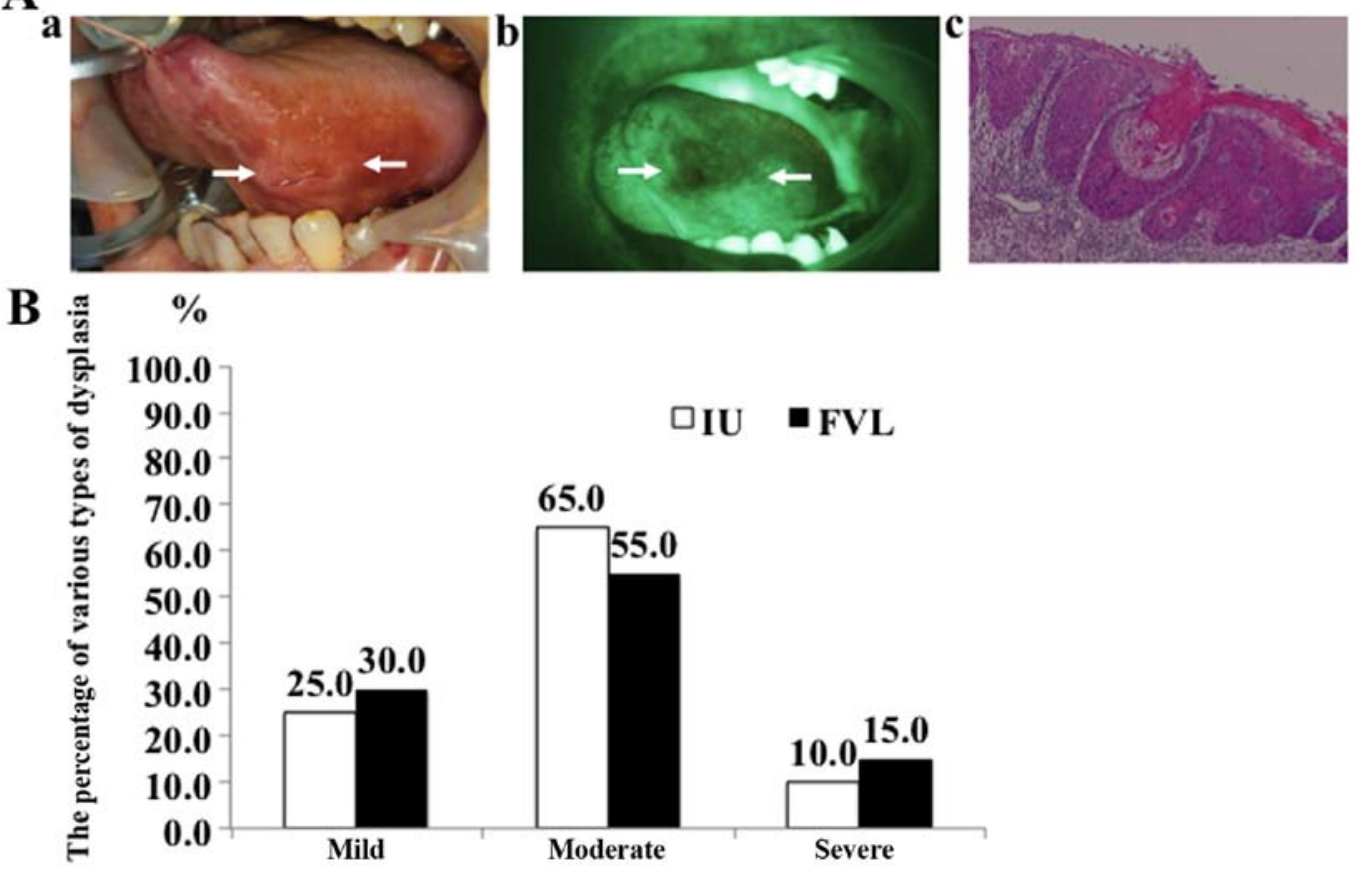

Figure 5. Comparison between iodine unstained area (IU) and FV loss (FVL). (A) A case of squamous cell carcinoma (SCC). Arrows indicate the region of IU (a) and FVL (b). The histology is shown in (c). (B) Clinical study concerning the comparison between FVL and IU. There were no normal epithelium cells in any of the FVL regions. Furthermore, the percentages of various types of dysplasia were almost equal when comparing the FVL (mild $30.0 \%$, moderate $55.0 \%$ and severe $15.0 \%$ ) and IU results (mild $25.0 \%$, moderate $65.0 \%$ and severe $10.0 \%$ ). 
Table III. Summary of the analyzed studies referring to visualization systems (VELscope).

\begin{tabular}{lcccc}
\hline Author (ref.) & Type of article & Samples & Sensitivity (\%) & Specificity (\%) \\
\hline Poh et al (10) & Crosssectional study & 20 & 97 & 94 \\
Kois and Truelove (12) & Case series & 4 & 98 & 100 \\
Balevi (15) & Opinion article & - & 98 & 100 \\
Ohnishi et al (Present study) & & 20 & 91 & 100 \\
\hline
\end{tabular}

in the normal pale green and appeared as dark green to black were classified as FVL.

Invasive SCC case. In the SCC image (Fig. 4D), the lesion appeared as a clear, dark area. This area was confirmed to be SCC. This assessment involved a comparison of the lesion site with both adjacent tissue and, as an anatomical control, with tissue on the contralateral side.

Pathology. As shown in Table II, of the 60 samples, 13 were FVR, and 49 were FVL. None of the 7 samples with a histological diagnosis of normal showed FVL, whereas $90 \%$ of precancerous lesions (dysplasia and CIS) and 93\% of invasive SCC showed FVL.

Comparison with another report. According to the BCCA, this system has a sensitivity of $98 \%$ and specificity of $100 \%$. Poh et al showed $97 \%$ sensitivity and $100 \%$ specificity. This was very similar to our results of $91 \%$ sensitivity and $100 \%$ specificity (Table III).

A clinical study concerning the comparison between FVL and iodine unstained area (IU). To clarify the usefulness of FV compared to vital staining with iodine, we investigated the surgical margin of early OSCC tissues and compared the FVL and IU (Fig. 5A). Twenty cases of T1 and early T2 OSCC were examined in this study. At the time of surgery, the surgeon outlined the boundary with vital staining with iodine and fluorescence visualization. With the operating room lights off, we examined the lesion and outlined the FVL, measured its size, and recorded data on the surgical sheet. Moreover, we used vital staining with iodine and made a comparison between FVL and IU and resected according to the wider boundary of the outline. As a result, the entire area by FVL showed various types of epithelial dysplasia (Fig. 5B). There were no normal epithelium cells in any of the FVL regions. Furthermore, the percentages of various types of dysplasia were almost equal between FVL (mild $30.0 \%$, moderate $55.0 \%$ and severe $15.0 \%$ ) and IU (mild $25.0 \%$, moderate $65.0 \%$, and severe $10.0 \%$ ). We considered that determining the surgical margin based on results of FV would not lead to oversurgery and could help prevent SPTs.

\section{Discussion}

The purpose of this study was to assess the accuracy of autofluorescence examination and to investigate the utility of autofluorescence as a diagnostic test to detect oral precancerous and early cancerous lesions in rat tongue carcinogenesis and patients. We therefore used the 4NQO rat model, which displays similar developmental morphology to human OSCC. The 4NQO rat model has been recognized to pass through various stages of OSCC, such as dysplasia, CIS and SCC.

FVL was observed in the majority of the dysplasia and CIS lesions that had a relatively higher risk of malignant transformation. Importantly, FVL was positive in all CIS cases, with sensitivity of $100 \%$. FVL was also observed in the majority of cases (85\%) that were histopathologically diagnosed as dysplasia. These results notably demonstrate the ability of the technique to detect high-risk lesions. Furthermore, using this 4 NQO rat model is the first such study.

In this study, we also investigated the expression of PCNA during 4NQO-induced carcinogenesis. The level of PCNA protein gradually increased with progression of carcinogenic transformation. Significant differences in PCNA expression were statistically demonstrated between the normal and dysplasia groups. Levels of protein remained high, especially after the period of dysplastic change. Higher expression was noted in the SCC cases. Furthermore the results of PCNA and FVL were correlated.

Moreover, we investigated the value of this device to delineate field change in autofluorescence around cancers by determining and comparing the histopathologic changes of margins that retained normal FV with those margins that showed FVL in the patients.

According to the BCCA, this system has a sensitivity of $98 \%$ and a specificity of $100 \%$ in discriminating between normal tissues and severe dysplasia, CIS or invasive carcinoma. Our results were almost the same with $91 \%$ sensitivity and $100 \%$ specificity. Thus, we conclude that the VELscope is useful in assessing surgical margins in patients with oral precancerous and early cancerous lesions. This makes it extremely valuable in surgical management.

Important progress has been made in understanding the mechanisms responsible for endogenous fluorescence from epithelial tissues and how this fluorescence changes with dysplastic progression (16-18). The fluorophores of interest here are those that excite in the blue spectrum and have properties that have been spectroscopically correlated with dysplastic progression. The reduced form of nicotinamide adenine dinucleotide (NADH) and the oxidized form of flavin adenine dinucleotide (FAD) are important fluorophores that are good indicators of cellular metabolism. It has been shown that the strength of fluorescence due to NADH increases with dysplastic progression, and that of FAD decreases $(18,19)$.

Based on the present study of the origins of fluorescence and its change with dysplastic progression, we believe that FVL associated with dysplastic progression in the current 
device is primarily due to breakdown of the collagen matrix and increased hemoglobin absorption. Secondary to these effects is increased dispersion in the epithelium, epithelial thickening, and a decrease in FAD concentration.

In the use of iodine staining to diagnose oral lesions, the iodine monad is absorbed by a spiral of one amylase which constitutes a normal chain portion of starch existing in the cytoplasm, and the reaction is visualized as a brown color (20). Sawataishi et al (21) reported that glycogen in cells of the esophageal mucosa react with iodine. This reaction does not occur in dysplastic mucosa, due to the lack of glycogen granules in the cytoplasm of these cells. Furthermore, ultrastructurally glycogen granules in cells of the esophageal mucosal epithelium decrease with the grade of epithelial dysplasia. Another study suggested that mild dysplastic epithelia that stain with iodine may be normal epithelia, whereas both moderate and severe dysplasia that are unstained with iodine may be malignant lesions (22). To clarify the usefulness of FV compared to vital staining with iodine, we investigated the surgical margins of early OSCC tissues and compared the results of FVL and IU. As a result, the entire area by FVL showed various types of epithelial dysplasia. There were no normal epithelium cells in any of the FVL regions. Furthermore, the percentages of various types of dysplasia were almost equal between the FVL and IU results.

These results suggest that this direct FV device has potential as a simple, cost-effective screening, biopsy guidance, and margin setting device for oral precancerous and early cancerous lesions.

\section{Acknowledgements}

This manuscript was edited for English language by Textcheck English consultants. Funding for this study was provided by Osaka Dental University (K.K.).

\section{References}

1. Chen YJ, Lin SC, Kao T, Chang CS, Hong PS, Shieh TM and Chang KW: Genome-wide profiling of oral squamous cell carcinoma. J Pathol 204: 326-332, 2004.

2. Pentenero M, Gandolfo S and Carrozzo M: Importance of tumor thickness and depth of invasion in nodal involvement and prognosis of oral squamous cell carcinoma: a review of the literature. Head Neck 27: 1080-1091, 2005.

3. Myers JN, Elkins T, Roberts D and Byers RM: Squamous cell carcinoma of the tongue in young adults: increasing incidence and factors that predict treatment outcomes. Otolaryngol Head Neck Surg 122: 44-51, 2000.

4. Choong N and Vokes E: Expanding role of the medical oncologist in the management of head and neck cancer. CA Cancer J Clin 58: 32-53, 2008 .

5. De VeldDCG, Witjes MJH,Sterenborg HJCM and RoodenburgJLN: The status of in vivo autofluorescence spectroscopy and imaging for oral oncology. Oral Oncol 41: 117-131, 2005.
6. Onizawa K, Saginoya H, Furuya Y, Yoshida H and Fukuda H: Usefulness of fluorescence photography for diagnosis of oral cancer. Int J Oral Maxillofac Surg 28: 206-210, 1999.

7. Onizawa K, Saginoya H, Furuya Y and Yoshida H: Fluorescence photography as a diagnostic method for oral cancer. Cancer Lett 108: 61-66, 1996.

8. Svistun E, Alizadeh-Naderi R, El-Naggar A, Jacob R, Gillenwater A and Richards-Kortum R: Vision enhancement system for detection of oral cavity neoplasia based on autofluorescence. Head Neck 26: 205-215, 2004.

9. Lane PM, Gilhuly T, Whitehead P, Zeng H, Poh CF, Ng S, Williams PM, Zhang L, Rosin MP and MacAulay CE: Simple device for the direct visualization of oral-cavity tissue fluorescence. J Biomed Opt 11: 024006, 2006.

10. Poh CF, Ng SP, Williams PM, Zhang L, Laronde DM, Lane P, Macaulay C and Rosin MP: Direct fluorescence visualization of clinically occult high-risk oral premalignant disease using a simple hand-held device. Head Neck 29: 71-76, 2007.

11. Qu J, MacAulay C, Lam S and Palcic B: Laser induced fluorescence spectroscopy at endoscopy: tissue optics, Monte Carlo modeling and in vivo measurements. Opt Eng 34: 3334-3343, 1995.

12. Kois JC and Truelove E: Detecting oral cancer: a new technique and case reports. Dent Today 25: 94-97, 96-97, 2006.

13. Poh CF, Zhang L, Anderson DW, Durham JS, Williams PM, Priddy RW, Berean KW, Ng S, Tseng OL, MacAulay C, et al: Fluorescence visualization detection of field alterations in tumor margins of oral cancer patients. Clin Cancer Res 12: 6716-6722, 2006.

14. Müller MG, Valdez TA, Georgakoudi I, Backman V, Fuentes C, Kabani S, Laver N, Wang Z, Boone CW, Dasari RR, et al: Spectroscopic detection and evaluation of morphologic and biochemical changes in early human oral carcinoma. Cancer 97: 1681-1692, 2003.

15. Balevi B: Evidence-based decision making: Should the general dentist adopt the use of the VELscope for routine screening for oral cancer? J Can Dent Assoc 73: 603-606, 2007.

16. Richards-Kortum R and Sevick-Muraca E: Quantitative optical spectroscopy for tissue diagnosis. Annu Rev Phys Chem 47: 555-606, 1996.

17. Drezek R, Sokolov K, Utzinger U, Boiko I, Malpica A, Follen M and Richards-Kortum R: Understanding the contributions of NADH and collagen to cervical tissue fluorescence spectra: modeling, measurements, and implications. J Biomed Opt 6: 385-396, 2001.

18. Drezek R, Brookner C, Pavlova I, Boiko I, Malpica A, Lotan R, Follen M and Richards-Kortum R: Autofluorescence microscopy of fresh cervical-tissue sections reveals alterations in tissue biochemistry with dysplasia. Photochem Photobiol 73: 636-641, 2001.

19. Pavlova I, Sokolov K, Drezek R, Malpica A, Follen M and Richards-Kortum R: Microanatomical and biochemical origins of normal and precancerous cervical autofluorescence using laser-scanning fluorescence confocal microscopy. Photochem Photobiol 77: 550-555, 2003.

20. Tsuboi S, Sato K and Nakajima K: Gendai no Seikagaku. Kanehara Publishing, Tokyo, pp55-56, 1992.

21. Sawataishi M, Karaki Y, Kawaguchi M, Saitoh M, Saeki T, Yamada A, Shimazaki K, Munakata S, Sakamoto T, Shinbo T, et al: The examination of the coloring mechanism of the human esophageal epithelium by Lugol's solution. J Jpn Bronchoesophagol Soc 40: 252-257, 1989.

22. Yokoo K, Noma H, Inoue T, Hashimoto S and Shimono M: Cell proliferation and tumor suppressor gene expression in iodine unstained area surrounding oral squamous cell carcinoma. Int $\mathbf{J}$ Oral Maxillofac Surg 33: 75-83, 2004. 